\title{
Anisotropic Fermi Surface and Quantum Limit Transport in High Mobility Three-Dimensional Dirac Semimetal $\mathrm{Cd}_{3} \mathrm{As}_{2}$
}

\author{
Yanfei Zhao, ${ }^{1,2}$ Haiwen Liu, ${ }^{1,2}$ Chenglong Zhang, ${ }^{1,2}$ Huichao Wang, ${ }^{1,2}$ Junfeng Wang, ${ }^{3}$ Ziquan Lin, ${ }^{3}$ Ying Xing, ${ }^{1,2}$ \\ Hong Lu, ${ }^{1,2}$ Jun Liu, ${ }^{4}$ Yong Wang, ${ }^{4}$ Scott M. Brombosz, ${ }^{5}$ Zhili Xiao, ${ }^{5,6}$ Shuang Jia, ${ }^{1,2, *}$ X. C. Xie, ${ }^{1,2,}$ and Jian Wang ${ }^{1,2, \sharp}$ \\ ${ }^{1}$ International Center for Quantum Materials, School of Physics, Peking University, Beijing 100871, China \\ ${ }^{2}$ Collaborative Innovation Center of Quantum Matter, Beijing 100871, China \\ ${ }^{3}$ Wuhan National High Magnetic Field Center, Huazhong University of Science and Technology, \\ Wuhan 430074, China \\ ${ }^{4}$ Center of Electron Microscopy, State Key Laboratory of Silicon Materials, \\ Department of Materials Science and Engineering, Zhejiang University, Hangzhou 310027, China \\ ${ }^{5}$ Materials Science Division, Argonne National Laboratory, Argonne, Illinois 60439, USA \\ ${ }^{6}$ Department of Physics, Northern Illinois University, DeKalb, Illinois 60115, USA \\ (Received 23 December 2014; revised manuscript received 7 August 2015; published 16 September 2015)
}

\begin{abstract}
Three-dimensional topological Dirac semimetals have a linear dispersion in 3D momentum space and are viewed as the 3D analogues of graphene. Here, we report angle-dependent magnetotransport on the newly revealed $\mathrm{Cd}_{3} \mathrm{As}_{2}$ single crystals and clearly show how the Fermi surface evolves with crystallographic orientations. Remarkably, when the magnetic field lies in the [112] or [44ī] axis, magnetoresistance oscillations with only single period are present. However, the oscillation shows double periods when the field is applied along the [1 $1 \overline{\mathbf{1}} 0]$ direction. Moreover, aligning the magnetic field at certain directions also gives rise to double period oscillations. We attribute the observed anomalous oscillation behavior to the sophisticated geometry of Fermi surface and illustrate a complete 3D Fermi surface with two nested anisotropic ellipsoids around the Dirac points. Additionally, a submillimeter mean-free path at $6 \mathrm{~K}$ is found in $\mathrm{Cd}_{3} \mathrm{As}_{2}$ crystals, indicating ballistic transport in this material. By measuring the magnetoresistance up to $60 \mathrm{~T}$, we reach the quantum limit $(n=1 \mathrm{Landau}$ level) at about $43 \mathrm{~T}$. These results improve the knowledge of the Dirac semimetal material $\mathrm{Cd}_{3} \mathrm{As}_{2}$ and also pave the way for proposing new electronic applications based on 3D Dirac materials.
\end{abstract}

DOI: 10.1103/PhysRevX.5.031037

\section{INTRODUCTION}

The three-dimensional topological Dirac semimetal, a new type of topological materials with a pair of nondegenerate 3D massless Weyl fermions, has become a rapidly growing field of research in condensed matter physics [1-13]. In analogy to two-dimensional Dirac points observed in graphene [14] and topological insulators [15-19], 3D Dirac semimetals possess bulk Dirac fermions with linear dispersion along three momentum directions [20].

Motivated by theoretical predications $[1,2]$, to date, $\mathrm{Na}_{3} \mathrm{Bi}$ [3] and $\mathrm{Cd}_{3} \mathrm{As}_{2}[4,5,10,21]$ have been identified to be 3D topological Dirac semimetals by angle-resolved photoemission spectroscopy (ARPES). More recently, STM experiments have revealed quasiparticle interference

\footnotetext{
"gwljiashuang@pku.edu.cn

xcxie@pku.edu.cn

*jianwangphysics@pku.edu.cn
}

Published by the American Physical Society under the terms of the Creative Commons Attribution 3.0 License. Further distribution of this work must maintain attribution to the author(s) and the published article's title, journal citation, and DOI.
Subject Areas: Condensed Matter Physics, Electronics, Materials Science

and the extended Dirac-like dispersion in $\mathrm{Cd}_{3} \mathrm{As}_{2}$ [6]. This unusual band dispersion also makes the electrons around the Fermi surface exhibit many unusual transport phenomena, such as strong quantum oscillations [8], ultrahigh mobility [9], and large magnetoresistance [9,12]. However, most magnetotransport measurements have been limited to study the quantum oscillations in one direction or by rotating the magnetic field only in a certain plane. The scarcity of the complete 3D Fermi surface analysis hinders an in-depth understanding of the physical properties in $\mathrm{Cd}_{3} \mathrm{As}_{2}$ single crystal. Therefore, it is highly desirable to demonstrate the transport property study at different magnetic field directions as well as the angular-dependent magnetotransport to reveal the complicated 3D Fermi surface in the $\mathrm{Cd}_{3} \mathrm{As}_{2}$ system. Further, the high magnetic field transport measurements to reveal the physics in the quantum limit of $\mathrm{Cd}_{3} \mathrm{As}_{2}$ are also highly pursued.

Here, we present a systematic study of the magnetotransport in a $\mathrm{Cd}_{3} \mathrm{As}_{2}$ single crystal and extend our study for the first time to the angular dependence of magnetoresistance in three independent directions as well as the high magnetic field experiments (up to $60 \mathrm{~T}$ ). The Shubnikov-de Haas $(\mathrm{SdH})$ oscillations signify a previously unknown Fermi 
surface with two nested ellipsoids, leading to a good understanding of its 3D Dirac nature and also providing a platform to explore exotic physical phenomena.

\section{RESULTS}

\section{A. Sample structure}

Single crystals of $\mathrm{Cd}_{3} \mathrm{As}_{2}$ are synthesized from a Cd-rich melt with the stoichiometry $\mathrm{Cd}_{8} \mathrm{As}_{3}$ in the evacuated quartz ampoule [22]. The $\mathrm{Cd}_{3} \mathrm{As}_{2}$ single crystal is examined by a FEI TITAN Cs-corrected cross-sectional STEM operating at $200 \mathrm{kV}$. Figure 1(a) shows the atomic layer-by-layer highangle annular dark field (HAADF) STEM image that manifests a high-quality single-crystal nature of a $\mathrm{Cd}_{3} \mathrm{As}_{2}$ sample. The XRD pattern shown in the inset with beam dimension of $0.5 \mathrm{~mm}$ diameter further confirms the singlecrystal property of our measured $\mathrm{Cd}_{3} \mathrm{As}_{2}$ sample. An optical image of the measured sample is shown in the inset of Fig. 1(d). The crystal is needlelike and grows preferentially along the $[1 \overline{\mathbf{1}} 0]$ direction (the length direction). The width direction is along $[44 \overline{\mathbf{1}}]$ and the largest facet of the crystal is the (112) plane. The standard four-probe method is used to measure the resistivity of the $\mathrm{Cd}_{3} \mathrm{As}_{2}$ sample on its (112) plane. Two indium or silver paste current electrodes $(I+$ and $I-)$ are pressed on both ends and across the entire width of the sample, so that the current can homogeneously go through the sample in the length direction [110]. The other two indium or silver paste electrodes are pressed on the crystal as voltage probes. The angular dependence of magnetoresistance is measured by rotating the sample in the (110) plane and the (112) plane, characterized by the angles $\theta$ and $\varphi$ illustrated in Fig. 1(b), respectively. The transport measurements are carried out in a PPMS-16T system (Quantum Design) and pulsed high magnetic field at Wuhan National High Magnetic Field Center.

\section{B. Ultrahigh mobility in $\mathbf{C d}_{3} \mathbf{A s}_{2}$ single crystal}

More than ten samples are studied. The data presented here are from three typical samples: samples 1, 2, and 3 . Figure 1(c) shows the resistivity of sample 1 as a function of temperature $(T)$. The resistivity decreases almost linearly when $T$ decreases from $300 \mathrm{~K}$ to about $6 \mathrm{~K}$ and then tends (a)
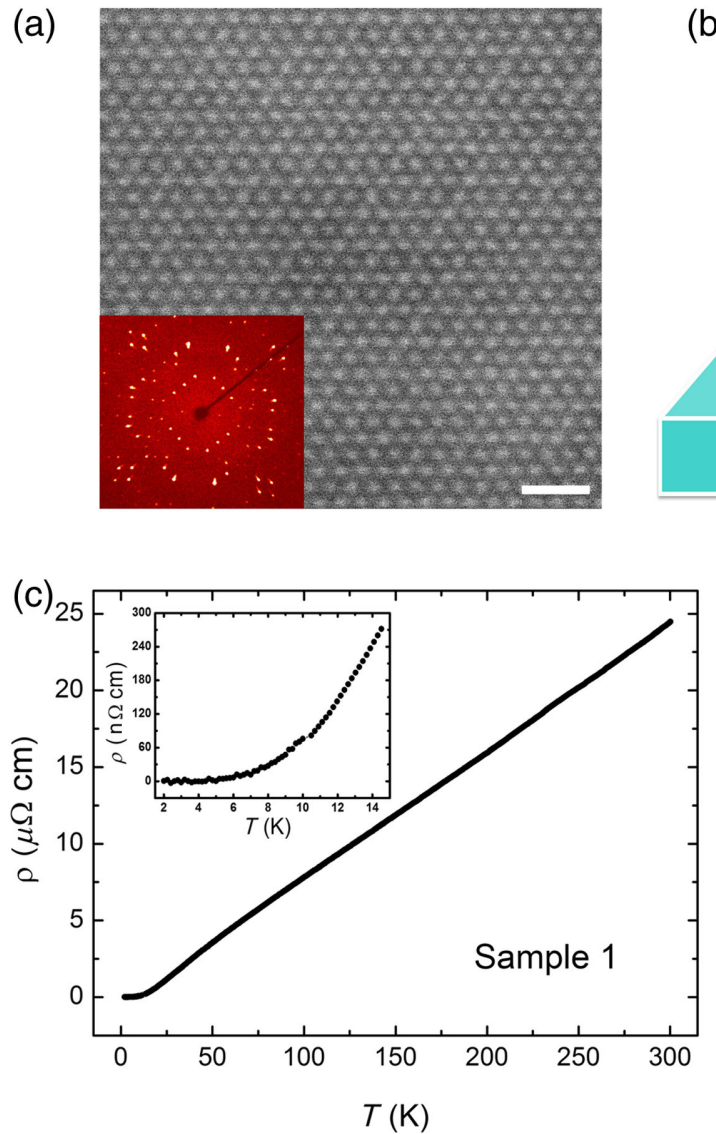

(b)
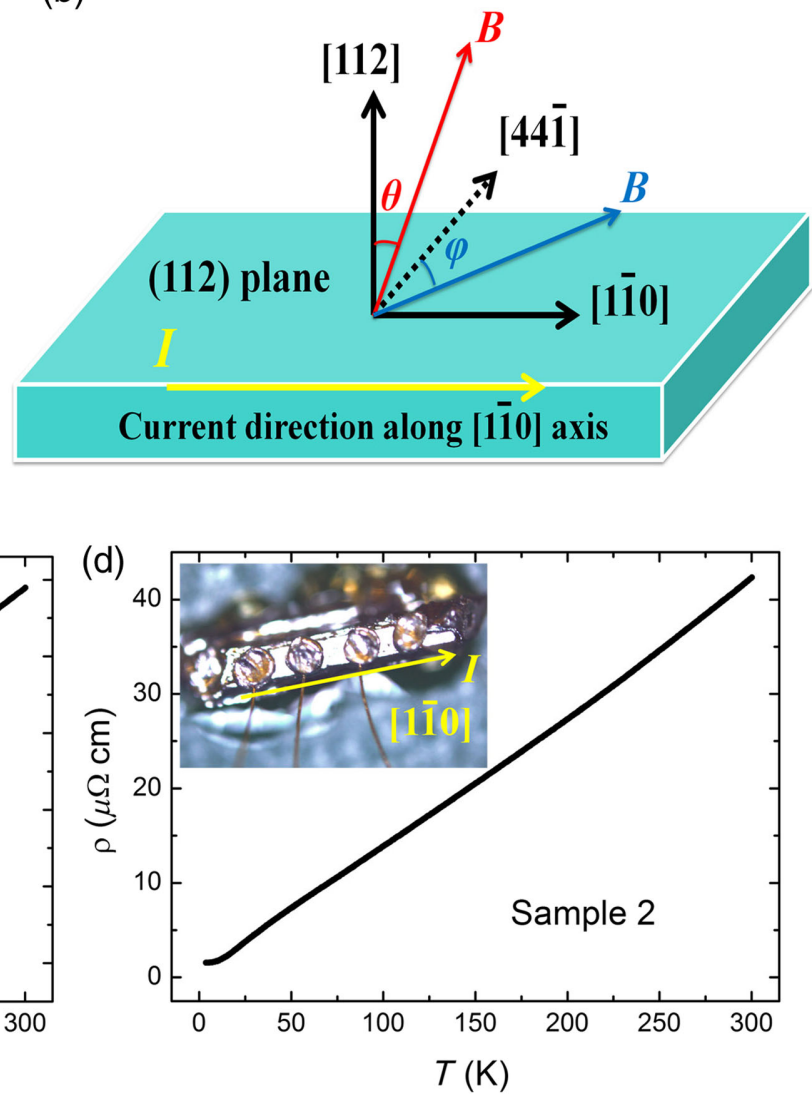

FIG. 1. Sample structure of $\mathrm{Cd}_{3} \mathrm{As}_{2}$ single crystal. (a) HAADF STEM image of $\mathrm{Cd}_{3} \mathrm{As}_{2}$ single crystal. Scale bar represents $1 \mathrm{~nm}$. Inset shows the crystal XRD image. (b) Schematic structure for the angular-dependent magnetotransport measurements in $\mathrm{Cd}_{3} \mathrm{As}_{2} \mathrm{system}$ Sample is rotated in (1) 10$)$ plane and (112) plane, characterized by the angle $\theta$ and $\varphi$, respectively. The sample size is not to scale. (c) Resistivity of sample 1 as a function of temperature. The inset shows the resistivity falling to quite low value about $11.6 \mathrm{n} \Omega \mathrm{cm}$ at $6 \mathrm{~K}$ and then reaching zero resistivity within instrumental resolution. (d) $\rho(T)$ behavior of sample 2 . The inset shows an optical image of the measured $\mathrm{Cd}_{3} \mathrm{As}_{2}$ sample. Standard four-probe method is used to measure the transport property. 
to be saturated. It displays a perfect linear metallic property with a large residual resistivity ratio $R R R=2120$ (the resistivity at room temperature over the resistivity at $6 \mathrm{~K}$ ). As shown in the inset of Fig. 1(c), the resistivity $\rho$ is quite low (about $11.6 \mathrm{n} \Omega \mathrm{cm}$ at $6 \mathrm{~K}$ ). Similar results have been reported before [9], suggesting the behavior is related to its long transport lifetime. More interestingly, when $T$ is lower than $6 \mathrm{~K}$, the resistivity oscillates near zero resistivity within the resolution of our measurement instrument (about $4.5 \mathrm{n} \Omega \mathrm{cm}$ ), which could be understood in terms of the quantum ballistic transport. Estimated from the $\mathrm{SdH}$ oscillations of sample 1, the carrier density is about $5.86 \times 10^{18} \mathrm{~cm}^{-3}$ (details are shown in Supplemental Material [23]). It is worth noting that the carrier density estimation from $\mathrm{SdH}$ might be 2-10 times smaller than the carrier density in the Hall measurement [9]. Therefore, the mobility is conservatively estimated to be $9.19 \times 10^{6}$ to $4.60 \times 10^{7} \mathrm{~cm}^{2} / \mathrm{V} \mathrm{s}$ and the mean-free path $l_{0}$ is about $0.25-1.25 \mathrm{~mm}$ at $6 \mathrm{~K}$, which is consistent with the previous transport study [9]. The high mobility and long mean-free path may shed new light on realizing various applications on future functional devices. Additionally, the linear metallic $\rho-T$ behavior is also observed in other samples, such as sample 2 shown in Fig. 1(d).

\section{3D anisotropic Fermi surface}

\section{Transport properties}

To investigate the 3D shapes of Fermi surfaces of $\mathrm{Cd}_{3} \mathrm{As}_{2}$, the analysis of $\mathrm{SdH}$ oscillations measured in three independent magnetic field directions, along [112], $[44 \overline{\mathbf{1}}]$, and $[1 \overline{\mathbf{1}} 0]$ axes $\left(B_{[112]}, B_{[44 \overline{\mathbf{1}}]}\right.$, and $\left.B_{[1 \mathbf{1} 0]}\right)$, are shown in Fig. 2. Figures 2(a)-(c) display the field dependencies of resistivity measured in three magnetic fields at different temperatures. Pronounced $\mathrm{SdH}$ oscillations are clearly visible around $4 \mathrm{~T}$ up to $50 \mathrm{~K}$, and then diminish at higher temperatures for all field directions. After subtracting a fourth-order polynomial background, the oscillatory component $\Delta \rho$ in three field directions is plotted in Figs. 2(d)-(f), respectively. The frequency of the $\mathrm{SdH}$ oscillations is extracted from the FFT analysis. Only one fundamental frequency is observed in both $B_{[112]}$ and $B_{[44 \overline{\mathbf{1}}]}$ directions with the period $B_{F[112]}=51 \mathrm{~T}$ and $B_{F[444 \overline{\mathbf{1}}]}=$ $41 \mathrm{~T}$, indicating a relatively simple but anisotropic Fermi surface in the two directions, and the Fermi surface in the $B_{[44 \overline{\mathbf{1}}]}$ direction is smaller than that measured in the $B_{[112]}$ direction. Interestingly, Fig. 2(f) illustrates a different set of $\mathrm{SdH}$ oscillations when the magnetic field is applied parallel to the $[1 \overline{1} 0]$ axis. Compared with the magnetoresistance (a)

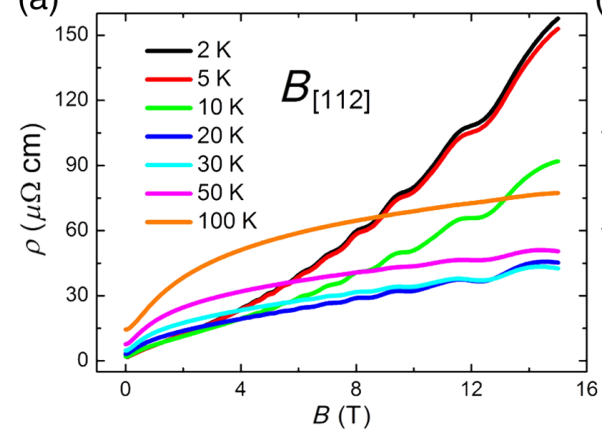

(d)

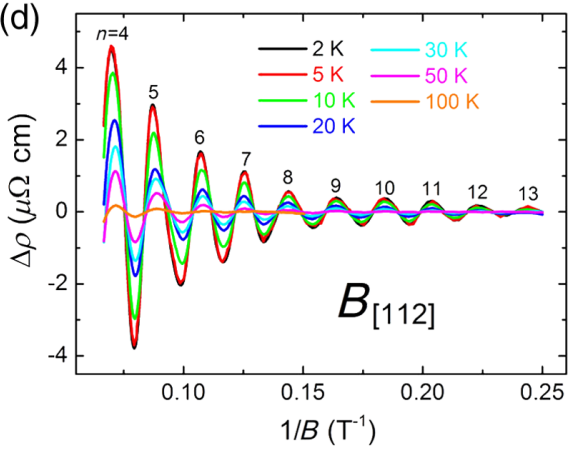

(b)
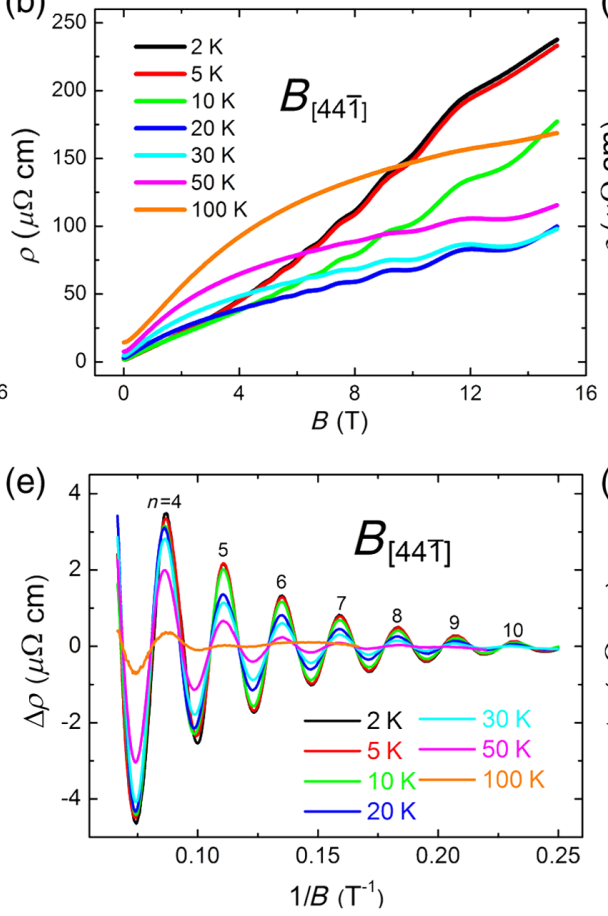

(c)

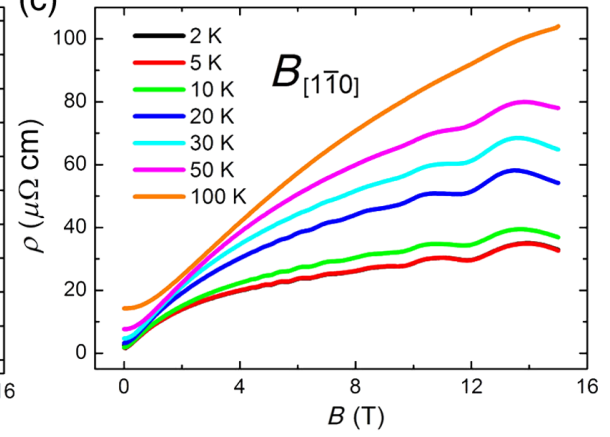

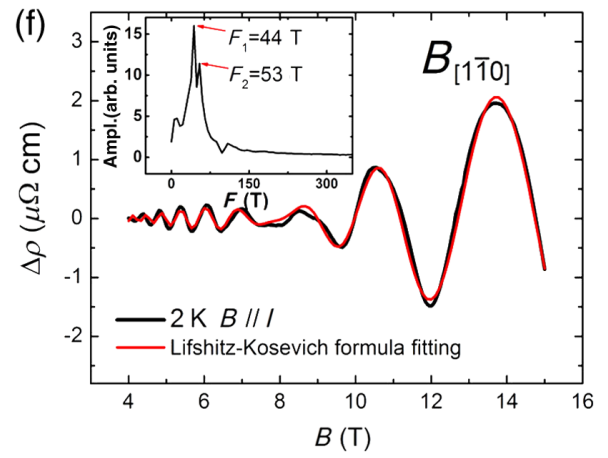

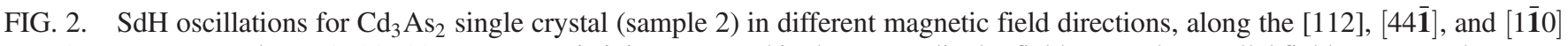
axes $\left(B_{[112]}, B_{[44 \overline{\mathbf{1}}]}\right.$, and $\left.B_{[1 \overline{\mathbf{1}} 0]}\right)$. (a)-(c) Magnetoresistivity measured in the perpendicular field $B_{[112]}$, the parallel fields $B_{[44 \mathbf{1}]}$, and $B_{[1 \overline{1} 0]}$ at different temperatures, respectively. (d),(e) The oscillatory component of $\Delta \rho$ extracted from $\rho$ by subtracting a fourth-polynomial background, as a function of $1 / B$ at various temperatures in $B_{[112]}$ and $B_{[44 \overline{1}]}$ directions. (f) The LK theory fitting to the $\Delta \rho(B)$ curve measured at $T=2 \mathrm{~K}$ in the parallel field $B_{[1 \overline{1} 0]}$ with two frequencies (red solid curve). Inset shows the FFT analysis with two frequencies in $B_{[1 \overline{1} 0]}$ direction. 


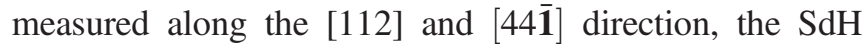
oscillations in the $B_{[1 \overline{1} 0]}$ direction are obviously weaker and nonperiodic. At around $8 \mathrm{~T}$, the oscillation seems to be suppressed and then appears again at about $10 \mathrm{~T}$. The inset of Fig. 2(f) shows the FFT of SdH oscillations in the $B_{[1 \overline{1} 0]}$ direction; two well-defined peaks appear at $F_{1}=44 \mathrm{~T}$ and $F_{2}=53 \mathrm{~T}$. We note that the resolution of our FFT analysis is sufficient enough to count the number of periodic oscillations. Actually, the resolution of our FFT results is similar to or even better than the FFT results previously reported in the topological insulators [24,25]. According to the Onsager relation, the frequency of the magnetoresistance oscillation is related to the external cross-sectional area $A_{k}$ of the Fermi surface in the momentum space: $F=(\hbar / 2 \pi e) A_{k}$, thus, gives two cross-sectional areas of Fermi surface perpendicular to the field $A_{F 1}=$ $4.210^{-3} \AA^{-2}$ and $A_{F 2}=5.1 \times 10^{-3} \AA^{-2}$, indicating a complicated Fermi surface.

In order to verify and analyze the $\mathrm{SdH}$ oscillations, we fit the entire oscillatory component with the standard Lifshitz-Kosevich (LK) theory for a 3D system [26-28]:

$$
\Delta \rho \propto \frac{\lambda}{\sinh \lambda} e^{-\lambda_{D}} \cos 2 \pi\left[\frac{F}{B}+\gamma-\delta\right],
$$

with $\lambda=2 \pi^{2} k_{B} T m^{*} / \hbar e B$ and $\lambda_{D}=2 \pi^{2} k_{B} T_{D} m^{*} / \hbar e B$, where $m^{*}$ is the cyclotron effective mass of the carriers and $T_{D}$ is the Dingle temperature. $\gamma$ is the phase related to the berry phase while $\delta$ is a phase shift determined by the dimensionality, taking the value $\delta= \pm 1 / 8$ for a 3D system $[27,29]$. Considering the two frequencies obtained in the $B_{[1 \overline{1} 0]}$ direction, we use two independent parameters $F_{1}$ and $F_{2}$ to fit the oscillation. As shown by the red solid line in Fig. 2(f), fitting to the LK theory yields two frequencies: $F_{1}=44.37 \mathrm{~T}$ and $F_{2}=52.85 \mathrm{~T}$. The two frequencies almost coincide with the FFT results $F_{1}=44 \mathrm{~T}$ and $F_{2}=53 \mathrm{~T}$, confirming the complex physics in this direction. On the other hand, the LK theory fitting to the oscillations in the $B_{[112]}$ and $B_{[44 \overline{\mathbf{1}}]}$ directions with a single frequency are shown in the Supplemental Material [23]. The fitting to the oscillation in the $B_{[112]}$ direction yields $F_{[112]}=54.13 \mathrm{~T}, T_{D}=36.93 \pm 1.5 \mathrm{~K}$, and $\gamma-\delta=0.3$, respectively. For the $B_{[44 \overline{1}]}$ case, the good fitting gives $F_{[444 \overline{\mathbf{1}}]}=42.6 \mathrm{~T}, T_{D}=33.54 \pm 2.5 \mathrm{~K}$, and $\gamma-\delta=0.2$. Both of the fitting results are consistent with the frequency identified from FFT spectra $\quad\left(F_{[112]}=51 \mathrm{~T}\right.$ and $\left.F_{[44 \overline{\mathbf{1}}]}=41 \mathrm{~T}\right)$. From the temperature dependence of the oscillation amplitude [Figs. 2(d) and 2(e)], we obtain the cyclotron effective mass $m_{[112]}^{*}=0.043 m_{e}$ and $m_{[44 \overline{\mathbf{1}}]}^{*}=$ $0.036 m_{e}$ ( $m_{e}$ is the free-electron mass). Therefore, taking into account all three different directions, the observed periods point to a 3D Fermi surface as a simple anisotropic

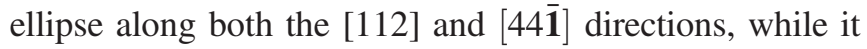

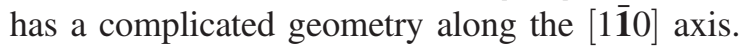

Moreover, to further understand the anisotropic 3D Fermi surface, analysis of the nontrivial Berry phase deduced from $\mathrm{SdH}$ oscillations is necessary. The Landau level (LL) fan diagram of the $B_{[112]}$ direction is plotted in Fig. 3(a). The maxima of $\rho$ are assigned to be the integer indices (solid circles) while the minima of $\rho$ are plotted by open circles in the diagram as half-integer indices [27]. A linear extrapolation of the index plot gives the intercept value close to 0.3. Considering the previous study [27], in a 3D system, the intercept of the index plot should be $0 \pm 1 / 8$ ( + for holes and - for electrons). In our system, the intercept is 0.3 , deviating from $\pm 1 / 8$, indicating the Fermi surface is an anisotropic ellipsoid instead of spherical with perfect $\pi$ Berry phase. Similarly, the LL fan diagram in the $B_{[44 \overline{\mathbf{1}}]}$ direction is shown in Fig. 3(b). As discussed previously, the intercept value obtained is about 0.2 , different from 0.3 yielded in the $B_{[112]}$ direction, also consistent with the assumption of the anisotropic Fermi surface in the $\mathrm{Cd}_{3} \mathrm{As}_{2}$ system. Figure 3(c) displays the magnetoresistance behavior measured in a pulsed high magnetic field normal to the (112) plane up to $54 \mathrm{~T}$ at various temperatures. The larger and more obvious $\mathrm{SdH}$ oscillations in high field provide an opportunity to further study the physics in the quantum limit. However, limited by the measurement resolution, the small oscillations in the lower field are hard to distinguish from noise. A similar plot based on the maxima and minima of $\rho$ versus the index $n$ is shown in Fig. 3(d). The intercept shifts to 0.38 instead of 0.3 deduced from the relatively low magnetic field. It suggests that the high magnetic field is needed to fix the intercept more reliably. It is worth noting that we reach the quantum limit in sample 3. More details are shown in Sec. II D.

To study the 3D Fermi surface of $\mathrm{Cd}_{3} \mathrm{As}_{2}$ systematically, the angular dependence of $\mathrm{SdH}$ oscillations is important to show the evolution of the Fermi surface changing in different directions. Figure 4(a) shows the angular-dependent oscillations after removing the polynomial background for the transverse rotation $(B \perp I)$ by varying the magnetic field angle $\theta$ tilting from the perpendicular field $\left(B_{[112]}\right)$ to the parallel field $\left(B_{[44 \overline{\mathbf{1}}]}\right)$. $\theta$ is the angle between the magnetic field and the [112] axis. Figure 4(b) shows the angular-dependent $\mathrm{SdH}$ oscillations for the transverse to longitudinal rotation in

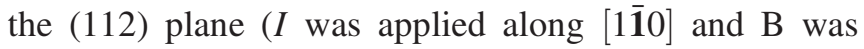
rotated from $[44 \overline{\mathbf{1}}]$ towards $[1 \overline{\mathbf{1}} 0]) . \varphi$ is the angle between the magnetic field and the $[44 \overline{\mathbf{1}}]$ axis. As shown in Table I, the FFT analysis indicates only one period feature

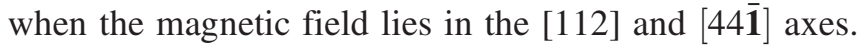
However, the system shows double period oscillations

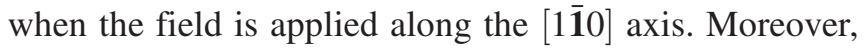
when changing the magnetic field angle $\theta$ and $\varphi$, two period oscillations also present. We attribute these anomalous $\mathrm{SdH}$ oscillations to the sophisticated geometry of the Fermi surface. (The corresponding FFT data are shown in the Supplemental Material [23].) 
(a)

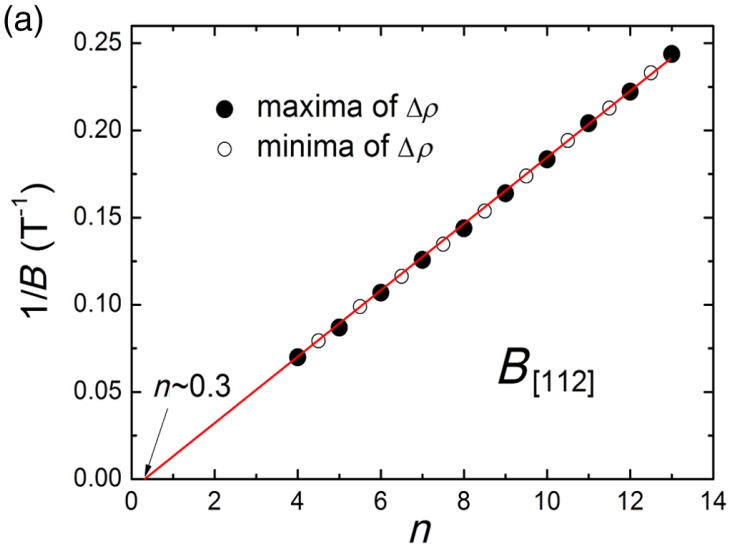

(c)

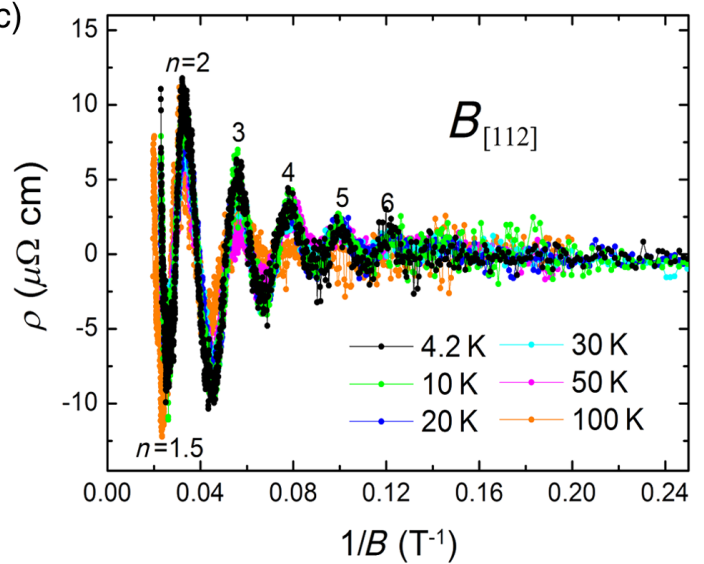

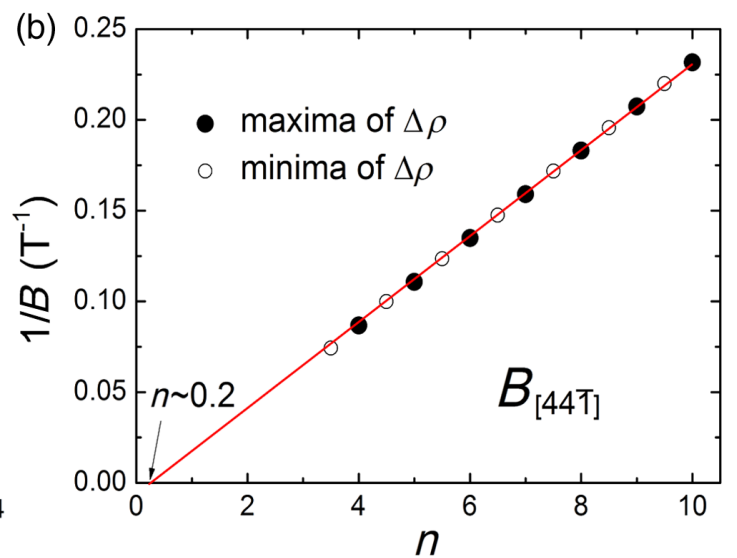

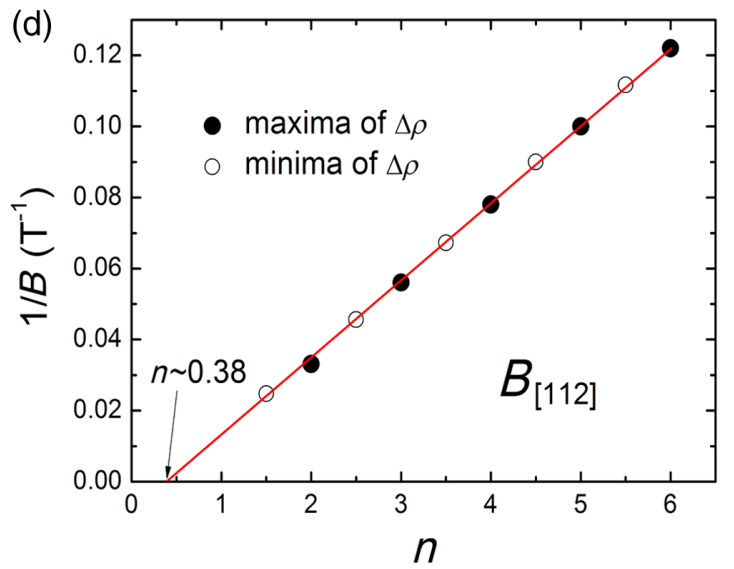

FIG. 3. Landau level index plots for $\mathrm{Cd}_{3} \mathrm{As}_{2}$ single crystal (sample 2) in $B_{[112]}$ and $B_{[44 \overline{1}]}$ directions. (a) Landau level index plot of the Fermi surface in the perpendicular field $B_{[112]}$. The maxima of $\Delta \rho$ are assigned to be the integer indices (solid circles) while the minima of $\Delta \rho$ are plotted by open circles as half-integer indices. The intercept is close to 0.3. (b) Landau level plot for oscillations in $\Delta \rho$ measured at $2 \mathrm{~K}$ in the parallel field $B_{[44 \overline{\mathbf{1}}]}$. Solid line is a linear fitting to the data, giving the intercept about 0.2 . (c) $\mathrm{SdH}$ oscillations measured in the pulsed high magnetic field in the perpendicular field $B_{[112]}$. (d) The index plot of $1 / B$ versus $n$ measured in pulsed high magnetic field. The intercept is about 0.38 .
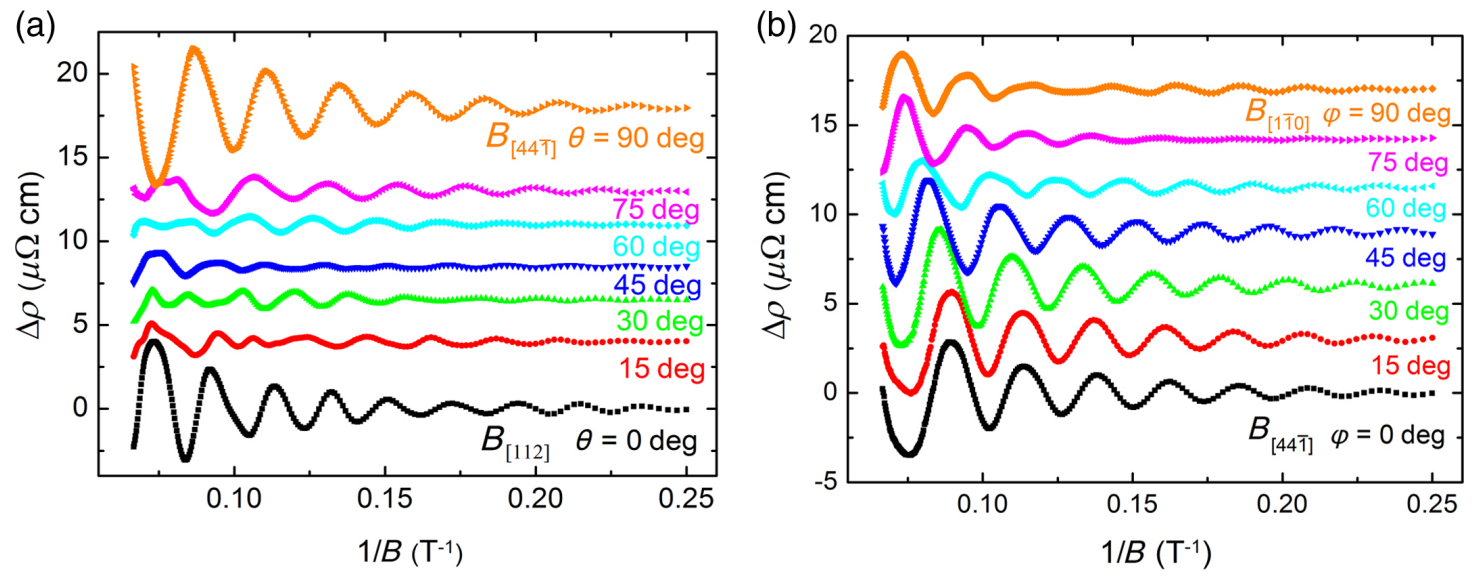

FIG. 4. Angular dependence of $\mathrm{SdH}$ oscillations at different magnetic field directions (sample 2). (a) $\mathrm{SdH}$ oscillations in $\Delta \rho$ for various

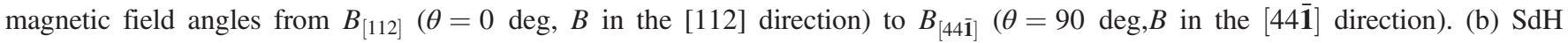
oscillations rotate from $B_{[44 \overline{\mathbf{1}}]}\left(\varphi=0 \mathrm{deg}, B\right.$ in the $[44 \overline{\mathbf{1}}]$ direction) to $B_{[1 \overline{\mathbf{1}} 0]}(\varphi=90 \mathrm{deg}, B$ in the $[1 \overline{\mathbf{1}} 0]$ direction). Figure $1(\mathrm{~b})$ depicts the definition of $\theta$ and $\varphi$. 
TABLE I. FFT analysis of $\mathrm{SdH}$ oscillations in various magnetic field directions.

\begin{tabular}{lccccccc}
\hline \hline $\begin{array}{l}\text { Rotation } \\
\text { angle }\end{array}$ & $\begin{array}{c}0 \\
\mathrm{deg}\end{array}$ & $\begin{array}{c}15 \\
\mathrm{deg}\end{array}$ & $\begin{array}{c}30 \\
\mathrm{deg}\end{array}$ & $\begin{array}{c}45 \\
\mathrm{deg}\end{array}$ & $\begin{array}{c}60 \\
\mathrm{deg}\end{array}$ & $\begin{array}{c}75 \\
\mathrm{deg}\end{array}$ & $\begin{array}{c}90 \\
\mathrm{deg}\end{array}$ \\
\hline$B_{[112]}$ rotate & $51 \mathrm{~T}$ & $44 \mathrm{~T}$ & $51 \mathrm{~T}$ & $51 \mathrm{~T}$ & $46 \mathrm{~T}$ & $43 \mathrm{~T}$ & $41 \mathrm{~T}$ \\
to $B_{[44 \overline{1}]}$ & & $55 \mathrm{~T}$ & $62 \mathrm{~T}$ & & & & \\
$B_{[44 \overline{1}]}$ rotate & $41 \mathrm{~T}$ & $41 \mathrm{~T}$ & $44 \mathrm{~T}$ & $44 \mathrm{~T}$ & $46 \mathrm{~T}$ & $45 \mathrm{~T}$ & $44 \mathrm{~T}$ \\
to $B_{[1 \overline{1} 0]}$ & & & & & $51 \mathrm{~T}$ & $52 \mathrm{~T}$ & $53 \mathrm{~T}$ \\
\hline \hline
\end{tabular}

\section{Theoretical analysis}

From pervious theoretical and experimental results [2,4], the Fermi surface is two anisotropic ellipsoids around the Dirac point, with Fermi momentum $k_{z}$ much larger than $k_{x}$ and $k_{y}$. Based on the Hall measurements in our $\mathrm{Cd}_{3} \mathrm{As}_{2}$ sample (see Fig. S7 in the Supplemental Material [23]), the carrier is $n$-type, indicating that the Fermi energy locates in the conduction band, which is similar to previous transport measurements [8,9] but different from several ARPES measurements [4,21]. Moreover, previous STM studies find that the Fermi energy locates in the conduction band and the Fermi energy is higher than the Lifshitz point [6]. Thus, when the Fermi energy goes beyond the Lifshitz saddle point, these two separate ellipsoids finally change into two nested ellipsoids. As shown in Figs. 5(c) and 5(d), the anomalous two-periods $\mathrm{SdH}$ oscillation in the $B_{[1 \overline{1} 0]}$ direction emerges when the maximum cross section of the Fermi surface passes through both the "neck" and the "belly" of two nested ellipsoids, which is similar to previous $\mathrm{SdH}$ oscillations in $\mathrm{Bi}-\mathrm{Sb}$ alloy [30] and $\mathrm{Te}$
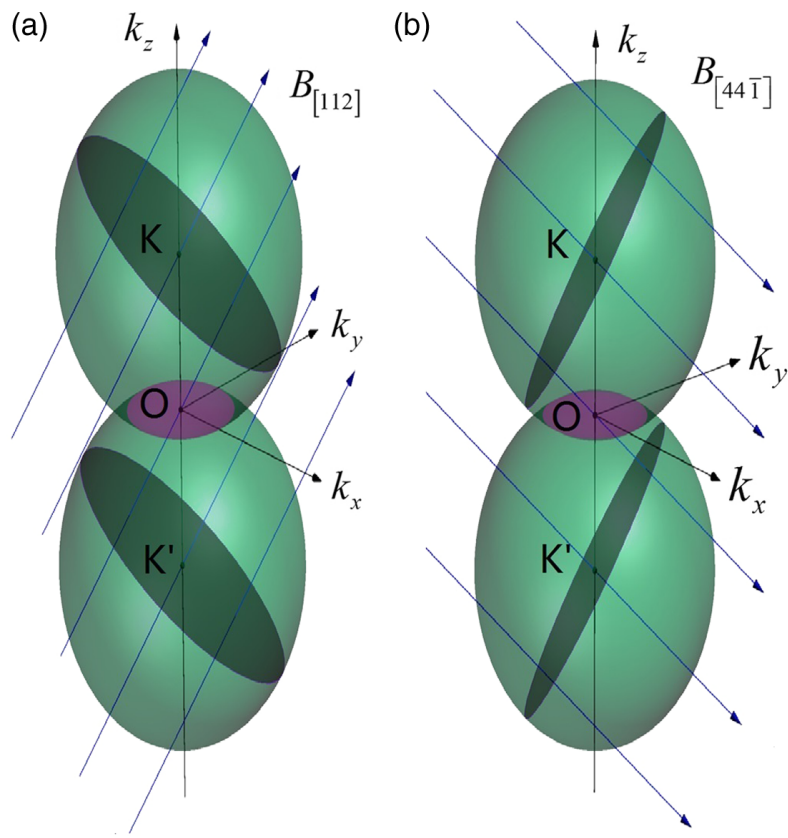

[31]. Although several ARPES measurements demonstrate that Fermi energy crosses multiple valence bands for the $p$-type system [4,21], the multiple bands are absent in our $n$-type system. For the other ARPES measurements $[5,10]$, when the Fermi energy locates in the conduction band, no obvious multiband features arise in these observations. Moreover, for the STM measurements [6], the Fermi energy also locates in the conduction band, and the second conduction band appears at about $300 \mathrm{meV}$ higher than the Fermi level (about $500 \mathrm{meV}$ higher than the Weyl point). Thus, in our measurement of the $n$-type system with a Fermi level about $200 \mathrm{meV}$ higher than the Weyl point, we think the multiband effect has no effect on the period of $\mathrm{SdH}$ oscillation. On the other hand, the possible $\mathrm{SdH}$ oscillation from the surface Fermi arc states can also be excluded. When the magnetic field is parallel to the (112) plane, the surface Fermi arc states have no effect on the $\mathrm{SdH}$ oscillations. Moreover, previous experimental studies have revealed the anisotropic $g$ factor in $\mathrm{Cd}_{3} \mathrm{As}_{2}$ with larger $g$ factor along the [001] direction [32]. However, this larger Zeeman coupling along the [001] direction cannot lead to the anomalous $\mathrm{SdH}$ oscillation when the magnetic field is along the $[1 \overline{\mathbf{1}} 0]$ direction. Furthermore, the observed anomalous beating pattern of the $\mathrm{SdH}$ oscillation possessing two frequencies in the FFT spectrum is inconsistent with the Zeeman splitting mechanism, while it usually originates from the double cross sections of the Fermi surface [33]. Taking into account the above factors, we conclude that the Fermi energy in our system locates slightly above the Lifshitz point, and the Fermi surface with nested ellipsoids shape results in one-period or

(c)

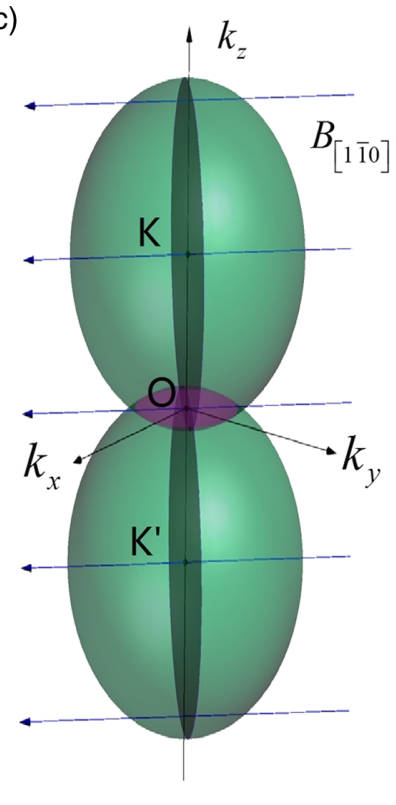

(d)

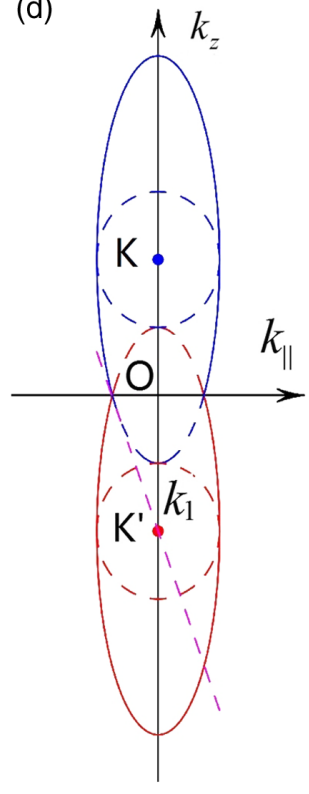

FIG. 5. Theoretical analysis of 3D nested anisotropic Fermi surface in $\mathrm{Cd}_{3} \mathrm{As}_{2}$. Panels (a)-(c) show the largest cross section of Fermi surface versus the magnetic field orientation. The Fermi surface is two nested anisotropic ellipsoids. Panel (d) shows the schematic plot of the Fermi surface for $B_{[1 \overline{1} 0]}$ direction with an overlapping region. The figures are not drawn to scale. 
two-periods $\mathrm{SdH}$ oscillation features dependent on the magnetic field orientation.

To further analyze the nested ellipsoid Fermi surface beyond the Lifshitz saddle point, we calculate the Fermi wave vectors at different directions. Based on the $\mathrm{SdH}$ periods $S_{F[112]}=51 \mathrm{~T}$ and $S_{F[44 \overline{\mathbf{1}}]}=41 \mathrm{~T}$ for the $B_{[112]}$ and $B_{[44 \overline{\mathbf{1}}]}$ directions, we obtain the mean momentum $k_{[112]}=$ $0.040 \AA^{-1}$ and $k_{[44 \overline{\mathbf{1}}]}=0.035 \AA^{-1}$, implying an anisotropic rather than a spherical Fermi surface, which is qualitatively consistent with ARPES results [4]. Next, we analyze the anomalous $\mathrm{SdH}$ oscillations observed in the angulardependent measurements shown in Table I. This anomalous $\mathrm{SdH}$ oscillation can be attributed to the nested ellipsoid Fermi surface beyond the Lifshitz saddle point. Firstly, for the $B_{[112]}$ and $B_{[44 \overline{\mathbf{1}}]}$ direction, the maximum cross section of the Fermi surface does not pass through the nested region [Figs. 5(a) and 5(b)], thus, giving only single period. When the magnetic field is twisted from [112] to $[44 \overline{\mathbf{1}}]$, the maximum cross sections of the Fermi surface penetrate into the nested region at certain angle range and form two nested ellipses, as shown in Fig. 5(d). Secondly,

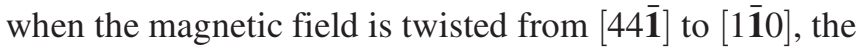
maximum cross section of the Fermi surface also forms two nested ellipses when the direction of the magnetic field is around the [1ㅣㅣㅇ direction [Figs. 5(c) and 5(d)]. The two nested ellipses lead to the anomalous two-period $\mathrm{SdH}$ oscillations in Table I. As shown in Fig. 5(d), the small dashed (red or blue) ellipse leads to the almost unchanged period $F_{1} \approx 44 \mathrm{~T}$, while the overlapping region corresponds to the period $F_{2} \approx 53 \mathrm{~T}$ shown in Table $\mathrm{I}$. Therefore, the geometry of the maximum cross sections in the Fermi surface can qualitatively interpret the anomalous $\mathrm{SdH}$ oscillations when tilting the magnetic field angle. (See the Supplemental Material for further analysis [23]).

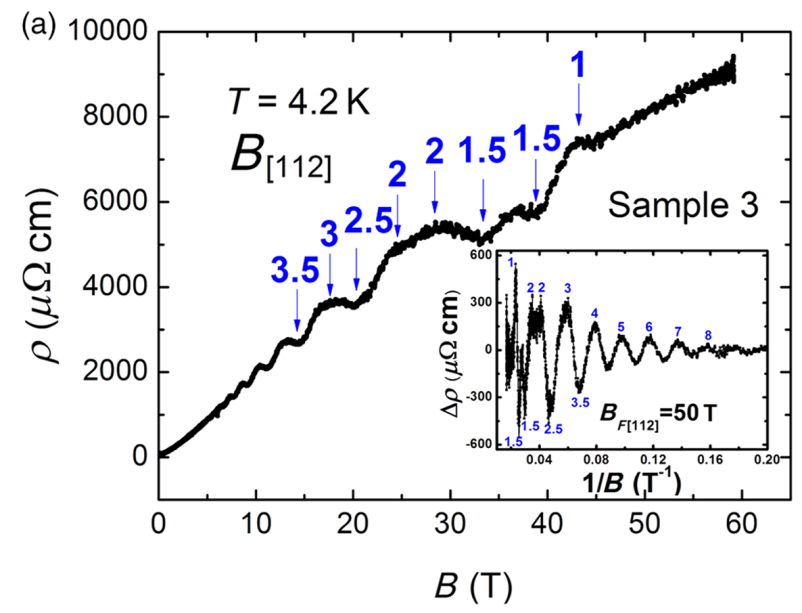

From the above analysis, we know that the Fermi energy is above the Lifshitz saddle point and the Fermi surface merges in certain momentum region. Thus, the topological correction from both Dirac points contribute to the Landau level intercept value, which makes the intercept value deviate from the theoretical value of $-1 / 8$ for electron type and results in different intercept values for different magnetic field orientations, which is consistent with the STM results [6]. In addition, comparing the Fermi surface with the previously reported Wigner-Seitz unit cell [2], we conclude that the Fermi surface in $\mathrm{Cd}_{3} \mathrm{As}_{2}$ is very large. Because of this large Fermi surface, the umklapp relation [34] can be satisfied and the umklapp electron-phonon scattering process plays a dominant role in the resistivity at low temperature in $\mathrm{Cd}_{3} \mathrm{As}_{2}$, which leads to $R \sim T \cdot N_{\mathrm{ph}}$, with $N_{\mathrm{ph}}$ denoting the number of phonons that satisfy the umklapp relation [34]. Moreover, due to the ultralarge unit cell of $\mathrm{Cd}_{3} \mathrm{As}_{2}$, low-energy optical phonon modes might exist in the system. Therefore, the umklapp processes and optical phonon modes can lead to an almost linear $R-T$ relation down to very low temperature, and the observed linear $R-T$ behavior in Figs. 1(c) and 1(d) deviates from the ordinary Bloch-Grüneisen law with $R \sim T^{5}$ and the electron-electron interaction induced $R \sim T^{2}$ law [34].

\section{Quantum limit of $\mathrm{Cd}_{3} \mathrm{As}_{2}$ single crystal: $\mathrm{SdH}$ oscillations with Zeeman splitting and linear magnetoresistance in the quantum limit}

We extend the $\mathrm{SdH}$ measurements to $60 \mathrm{~T}$ to explore the quantum limit. According to previous theory [35], a quantum linear magnetoresistance would be expected to occur in the quantum limit in a gapless semiconductor with linear energy dispersion, where all the carriers occupy the lowest Landau Level. Figure 6(a) displays the SdH oscillations of sample 3 in the $B_{[112]}$ direction measured in the

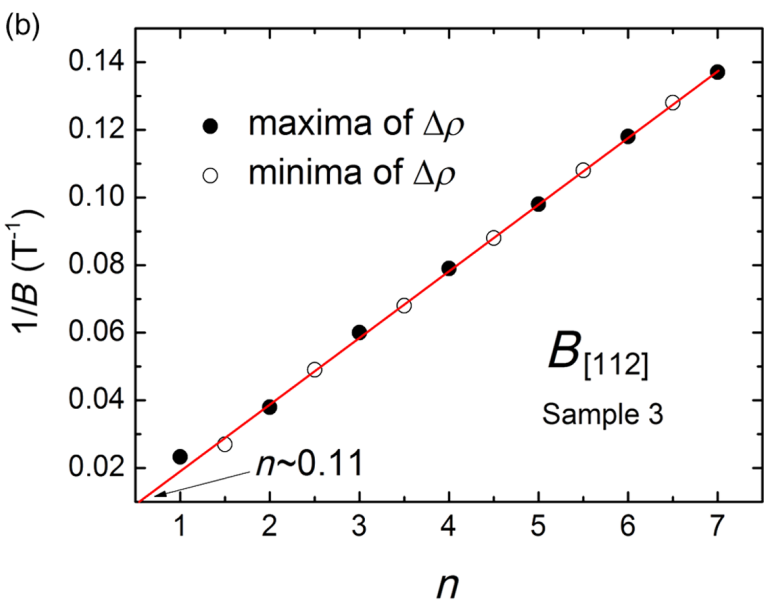

FIG. 6. High magnetic field transport measurement of Sample 3 up to 60 T. (a) Magnetoresistivity measured in the perpendicular field $B_{[112]}$ at different temperatures at $T=4.2 \mathrm{~K}$ up to $60 \mathrm{~T}$. Zeeman splitting features can be observed at higher magnetic field. Inset shows the SdH oscillations after subtracting the polynomial background. (b) Landau level index plot. The $n=1$ Landau Level is reached at $B=43 \mathrm{~T}$. Quantum linear magnetoresistance is observed at the field higher than $43 \mathrm{~T}$. 
pulsed high magnetic field at $T=4.2 \mathrm{~K}$. At low field $(B<15 \mathrm{~T})$, the magnetoresistance shows perfect $\mathrm{SdH}$ oscillations, while in the relative higher field $(15<B<43 \mathrm{~T})$ it exhibits the $\mathrm{SdH}$ oscillations with Zeeman splitting. Careful investigation shows obvious Zeeman splitting around the $n=2$ peak and the $n=1.5$ dip. The Landau level index demonstrates that the system reaches the quantum limit at about $43 \mathrm{~T}$. With increasing the magnetic field beyond the quantum limit $(B>43 \mathrm{~T})$, the magnetoresistance presents a linear behavior that is consistent with the theoretical consideration [35]. After subtracting the polynominal background [the inset of Fig. 6(a)], the SdH oscillations with the Zeeman splitting feature can be clearly observed. By analyzing the FFT of the oscillation, we yield a single frequency $F_{[112]}=50 \mathrm{~T}$. Thus, comparing with sample 2, the relatively small Fermi surface and the remarkable Zeeman splitting in sample 3 might be the reason for the apparent quantum limit at $B=$ $43 \mathrm{~T}$ in sample 3. Further studies are needed to uncover the exact cause. Moreover, by considering a similar anisotropic Fermi surface as sample 2, the carrier density can be estimated as $n_{\mathrm{SdH}}=5.19 \times 10^{18} \mathrm{~cm}^{-3}$. The Landau level index plot based on the positions of maxima and minima in $\rho$ as a function of $1 / B$ is shown in Fig. 6(b), with the intercept at about 0.11 . When approaching the quantum limit of $n=1$, the maxima (or minima) point shows small deviations from the fitting line, which originates from the anomalous oscillation in the 3D Dirac system around the quantum limit [36].

\section{CONCLUSIONS}

In summary, we report pronounced $\mathrm{SdH}$ oscillations measured along three different directions in high-quality $\mathrm{Cd}_{3} \mathrm{As}_{2}$ single crystals and extend our study to the angulardependent magnetotransport and in the high magnetic field up to the quantum limit. By analyzing the $\mathrm{SdH}$ oscillations in different magnetic field orientations, we obtain a complete 3D Fermi surface with two nested ellipsoids. Furthermore, we present that the changing of the angular-dependent oscillation periods is essentially due to the complicated nested 3D Fermi surface. In addition, the submillimeter scale mean-free path and ballistic transport region as well as the quantum limit are demonstrated in a $\mathrm{Cd}_{3} \mathrm{As}_{2}$ single crystal. These results intensify the previous knowledge of the Dirac semimetal material $\mathrm{Cd}_{3} \mathrm{As}_{2}$, offer a better understanding of existing 3D Dirac semimetals, and reveal the potential of application in topological electronic devices.

\section{ACKNOWLEDGMENTS}

We acknowledge Chong Wang, Yuan Li, Tian Qian, and Hong Ding for their help in Laue measurements and we thank Liang Li and Zhengcai Xia for helpful discussions about the pulsed magnetic field measurements. This work was financially supported by National Basic Research
Program of China (Grants No. 2013CB934600, No. 2015CB921102, and No. 2012CB921300), the National Natural Science Foundation of China (No. 11222434, No. 11534001, and No. 11174007), and the Research Fund for the Doctoral Program of Higher Education (RFDP) of China. S. M. B. and Z. L.X. at Argonne was supported by the U.S. Department of Energy, Office of Science, Basic Energy Sciences, Materials Sciences and Engineering Division.

Y.Z. and H. L contributed equally to this work.

[1] Z. J. Wang, Y. Sun, X.-Q. Chen, C. Franchini, G. Xu, H. M. Weng, X. Dai, and Z. Fang, Dirac Semimetal and Topological Phase Transitions in $\mathrm{A}_{3} \mathrm{Bi}(A=\mathrm{Na}, K, R b)$, Phys. Rev. B 85, 195320 (2012).

[2] Z. J. Wang, H. M. Weng, Q. S. Wu, X. Dai, and Z. Fang, Three-Dimensional Dirac Semimetal and Quantum Transport in $\mathrm{Cd}_{3} \mathrm{As}_{2}$, Phys. Rev. B 88, 125427 (2013).

[3] Z. K. Liu, B. Zhou, Y. Zhang, Z. J. Wang, H. M. Weng, D. Prabhakaran, S.-K. Mo, Z. X. Shen, Z. Fang, X. Dai, Z. Hussain, and Y. L. Chen, Discovery of a Three-Dimensional Topological Dirac Semimetal, $\mathrm{Na}_{3} \mathrm{Bi}$, Science 343, 864 (2014).

[4] Z. K. Liu, J. Jiang, B. Zhou, Z. J. Wang, Y. Zhang, H. M. Weng, D. Prabhakaran, S.-K. Mo, H. Peng, P. Dudin, T. Kim, M. Hoesch, Z. Fang, X. Dai, Z. X. Shen, D. L. Feng, Z. Hussain, and Y. L. Chen, A Stable Three-Dimensional Topological Dirac Semimetal $\mathrm{Cd}_{3} \mathrm{As}_{2}$, Nat. Mater. 13, 677 (2014).

[5] M. Neupane, S.-Y. Xu, R. Sankar, N. Alidoust, G. Bian, C. Liu, I. Belopolski, T.-R. Chang, H.-T. Jeng, H. Lin, A. Bansil, F. C. Chou, and M. Z. Hasan, Observation of a Three-Dimensional Topological Dirac Semimetal Phase in High-Mobility $\mathrm{Cd}_{3} \mathrm{As}_{2}$, Nat. Commun. 5, 3786 (2014).

[6] S. Jeon, B. B. Zhou, A. Gyenis, B. E. Feldman, I. Kimchi, A. C. Potter, Q. D. Gibson, R. J. Cava, A. Vishwanath, and A. Yazdani, Landau Quantization and Quasiparticle Interference in the Three-Dimensional Dirac Semimetal $\mathrm{Cd}_{3} \mathrm{As}_{2}$, Nat. Mater. 13, 851 (2014).

[7] Z. Zhu and J.E. Hoffman, Condensed-Matter Physics: Catching Relativistic Electrons, Nature (London) 513, 319 (2014).

[8] L. P. He, X. C. Hong, J. K. Dong, J. Pan, Z. Zhang, J. Zhang, and S. Y. Li, Quantum Transport in the Three-Dimensional Dirac Semimetal $\mathrm{Cd}_{3} \mathrm{As}_{2}$, Phys. Rev. Lett. 113, 246402 (2014).

[9] L. Tian, Q. Gibson, M. N. Ali, M. Liu, R. J. Cava, and N. P. Ong, Ultrahigh Mobility and Giant Magnetoresistance in $\mathrm{Cd}_{3} \mathrm{As}_{2}$ : Protection from Backscattering in a Dirac Semimetal, Nat. Mater. 14, 280 (2015).

[10] S. Borisenko, Q. Gibson, D. Evtushinsky, V. Zabolotnyy, B. Büchner, and R. J. Cava, Experimental Realization of a Three-Dimensional Dirac Semimetal, Phys. Rev. Lett. 113, 027603 (2014).

[11] Q. D. Gibson, L. M. Schoop, L. Muechler, L. S. Xie, M. Hirschberger, N.P. Ong, R. Car, and R. J. Cava, ThreeDimensional Dirac Semimetals: Design Principles and Predictions of New Materials, Phys. Rev. B 91, 205128 (2015). 
[12] J. Y. Feng, Y. Pang, D. S. Wu, Z. J. Wang, H. M. Weng, J. Q. Li, Z. Fang, Y. G. Shi, and L. Lu, Large Linear Magnetoresistance in Dirac Semimetal $\mathrm{Cd}_{3} \mathrm{As}_{2}$ with Fermi Surfaces Close to the Dirac Points, Phys. Rev. B 92, 081306 (2015).

[13] S. Zhang et al., Breakdown of Three-Dimensional Dirac Semimetal State in Pressurized $\mathrm{Cd}_{3} \mathrm{As}_{2}$, Phys. Rev. B 91, 165133 (2015).

[14] K. S. Novoselov, A. K. Geim, S. V. Morozov, D. Jiang, M. I. Katsnelson, I. V. Grigorieva, S. V. Dubonos, and A. A. Firsov, Two-Dimensional Gas of Massless Dirac Fermions in Graphene, Nature (London) 438, 197 (2005).

[15] M.Z. Hasan and C. L. Kane, Colloquium: Topological Insulators, Rev. Mod. Phys. 82, 3045 (2010).

[16] X.-L. Qi and S.-C. Zhang, Topological Insulators and Superconductors, Rev. Mod. Phys. 83, 1057 (2011).

[17] H. J. Zhang, C.-X. Liu, X.-L. Qi, X. Dai, Z. Fang, and S.-C. Zhang, Topological Insulators in $\mathrm{Bi}_{2} \mathrm{Se}_{3}, \mathrm{Bi}_{2} \mathrm{Te}_{3}$ and $\mathrm{Sb}_{2} \mathrm{Te}_{3}$ with a Single Dirac Cone on the Surface, Nat. Phys. 5, 438 (2009).

[18] Y. L. Chen, J. G. Analytis, J.-H. Chu, Z. K. Liu, S.-K. Mo, X. L. Qi, H. J. Zhang, D. H. Lu, X. Dai, Z. Fang, S. C. Zhang, I. R. Fisher, Z. Hussain, and Z.-X. Shen, Experimental Realization of a Three-Dimensional Topological Insulator, $\mathrm{Bi}_{2} \mathrm{Te}_{3}$, Science 325, 178 (2009).

[19] Y. F. Zhao, H. W. Liu, X. Guo, Y. Jiang, Y. Sun, H. C. Wang, Y. Wang, H.-D. Li, M.-H. Xie, X.-C. Xie, and J. Wang, Crossover from $3 D$ to $2 D$ Quantum Transport in $\mathrm{Bi}_{2} \mathrm{Se}_{3} / \mathrm{In}_{2} \mathrm{Se}_{3}$ Superlattices, Nano Lett. 14, 5244 (2014).

[20] B.-J. Yang and N. Nagaosa, Classification of Stable ThreeDimensional Dirac Semimetals with Nontrivial Topology, Nat. Commun. 5, 4898 (2014).

[21] H. M. Yi et al., Evidence of Topological Surface State in Three-Dimensional Dirac Semimetal $\mathrm{Cd}_{3} \mathrm{As}_{2}$, Sci. Rep. 4, 6106 (2014).

[22] M. N. Ali, Q. Gibson, S. Jeon, B. B. Zhou, A. Yazdani, and R. J. Cava, The Crystal and Electronic Structures of $\mathrm{Cd}_{3} \mathrm{As}_{2}$, the Three-Dimensional Electronic Analogue of Graphene, Inorg. Chem. 53, 4062 (2014).

[23] See Supplemental Material at http://link.aps.org/ supplemental/10.1103/PhysRevX.5.031037 for more details.
[24] K. Eto, Z. Ren, A. A. Taskin, K. Segawa, and Y. Ando, Angular-Dependent Oscillations of the Magnetoresistance in $\mathrm{Bi}_{2} \mathrm{Se}_{3}$ due to the Three-Dimensional Bulk Fermi Surface, Phys. Rev. B 81, 195309 (2010).

[25] A. A. Taskin, Z. Ren, S. Sasaki, K. Segawa, and Y. Ando, Observation of Dirac Holes and Electrons in a Topological Insulator, Phys. Rev. Lett. 107, 016801 (2011).

[26] D. X. Qu, Y. S. Hor, J. Xiong, R. J. Cava, and N. P. Ong, Quantum Oscillations and Hall Anomaly of Surface States in the Topological Insulator $\mathrm{Bi}_{2} \mathrm{Te}_{3}$, Science 329, 821 (2010).

[27] H. Murakawa, M. S. Bahramy, M. Tokunaga, Y. Kohama, C. Bell, Y. Kaneko, N. Nagaosa, H. Y. Hwang, and Y. Tokura, Detection of Berry's Phase in a Bulk Rashba Semiconductor, Science 342, 1490 (2013).

[28] C. Bell, M. S. Bahramy, H. Murakawa, J. G. Checkelsky, R. Arita, Y. Kaneko, Y. Onose, M. Tokunaga, Y. Kohama, N. Nagaosa, Y. Tokura, and H. Y. Hwang, Shubnikov-de Haas Oscillations in the Bulk Rashba Semiconductor BiTeI, Phys. Rev. B 87, 081109 (2013).

[29] M. Novak, S. Sasaki, K. Segawa, and Y. Ando, Large Linear Magnetoresistance in the Dirac Semimetal TlBiSSe, Phys. Rev. B 91, 041203 (2015).

[30] M. Y. Lavrenyuk, N. Y. Minina, and A. M. Savin, Formation of a Dumbbell Fermi Surface and Intraband Magnetic Breakdown due to Uniaxial Compression of the Alloy $\mathrm{Bi}_{0.78} \mathrm{Sb}_{0.22}$, JETP Lett. 46, 282 (1987).

[31] C. Guthmann and J. M. Thuillier, Fermi Surface of Tellurium, Phys. Status Solidi (b) 38, 635 (1970).

[32] F. A. P. Blom, J. W. Cremers, J. J. Neve, and M. J. Gelten, Anisotropy of the Electronic $g *$-Factor in Cadmium Arsenide, Solid State Commun. 33, 69 (1980).

[33] G. Landwehr and Ė. I. Rashba. Landau Level Spectroscopy (North-Holland, Amsterdam, 1991), Vol. 2.

[34] A. A. Abrikosov, Fundamentals of the Theory of Metals (North-Holland, Amsterdam, 1988), Vol. 1.

[35] A. A. Abrikosov, Quantum Magnetoresistance, Phys. Rev. B 58, 2788 (1998).

[36] P.E. Ashby and J. P. Carbotte, Theory of Magnetic Oscillations in Weyl Semimetals, Eur. Phys. J. B 87, 92 (2014). 